\title{
Histological Observation Related to the Use of Laser and Ultrasound on Bone Fracture Healing
}

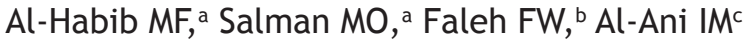 \\ aDepartment of Anatomy, College of Medicine, Al-Nahrain University, Baghdad, IRAQ. \\ ${ }^{b}$ College of Medical Engineering, Al-Nahrain University, Baghdad IRAQ. \\ 'Department of Basic Medical Science, Kulliyyah of Medicine, International Islamic University Malaysia, \\ Kuantan, Pahang, MALAYSIA.
}

\begin{abstract}
Objective: To study the effect of both laser and ultrasound radiation on bone fracture healing process. Materials and Methods: Nd:YAG laser (1064 nm wavelength, $135 \mathrm{~mW}$ power, 16 joules energy) and ultrasound (1 $\mathrm{MHz}$ frequency, $50 \mathrm{~mW} / \mathrm{cm}^{2}$ power intensity) were used in this work. Fifteen mature, male, albino rats, were divided into three groups and subjected to a partial fracture on the lateral aspect of femur by a sharp blade. The first group of these animals served as control group. The second group was illuminated by the $\mathrm{Nd}$ :YAG laser for two minutes; the first dose was given immediately after surgical fracture induction; the other doses were given on days two, three, six and then one dose weekly for the next three weeks while the third group were treated by the addition of the CW ultrasound perpendicular to the laser treatment in the second group. Results: The present study showed that ultrasound increases the penetration of laser power through the tissue. The histological assessments at day 28 after the fracture of first group showed incomplete healing of the bone with disfiguration and disarrangement of Haversian system and the periosteum was not yet well developed. Treatment with laser showed irregularity and lack of Haversian system formation in bone healing of the second group. The laser and ultrasound treated group (third group) expressed a complete healing at the site of fracture with a complete layer of periosteum and a well arranged Haversian system. Conclusion: Combination of laser and ultrasound in therapy can enhance healing process of a fractured bone more than laser therapy alone, as ultrasound increases the depth of laser penetration in tissue.
\end{abstract}

KEYWORDS: Bone, Fracture, Laser, Ultrasound.

\section{INTRODUCTION}

Tissue healing is a complex process that involves local and systemic responses. 1 Wound healing is a dynamic process of restoring cellular structures and tissue layers. The process of wound healing involves several types of cells; enzymes; growth factors and other substances. Bone healing process is slower than that of soft tissues and differs from it because of its morphology and composition. ${ }^{1}$ Healing of tendons and other dense connective tissues take as long as 6 - 8 weeks. ${ }^{2}$

Low-level laser therapy (LLLT) is a treatment refers to the use of red-beam or near-infrared lasers with a wavelength between 600 and 1000nm power from 5-500 milliwatts to stimulate the repair or reduction of pain of a wide variety of body parts. These types

\section{Corresponding author:}

Professor Imad M. Al-Ani,

Department of BMS,

Kulliyyah of Medicine,

International Islamic University,

P. O. Box 141, 25710 Kuantan, Pahang MALAYSIA.

Tel. +60179776014.

E-Mail: imad_alani@yahoo.com of lasers have been advocated for use in a wide range of medical conditions encompassing musculoskeletal conditions such as chronic low back pain, ${ }^{3}$ wound healing ${ }^{4}$ and acute and chronic Achilles tendinitis. ${ }^{5}$ LLLT has stimulatory effects on bone cell proliferation ${ }^{6}$ and gene expression ${ }^{7}$ and can be used to enhance bone repair at cellular and tissue levels. ${ }^{8}$ Laser effects on tissues may be attributed to thermal and non thermal effects; the non thermal effects of laser beams on tissue include photodynamic therapy and photobiostimulation therapy. The beneficial biological effects of laser light therapy on tissues can be summarized as to accelerate tissue repair and cell growth, improve vascular activity and increase metabolic activity. ${ }^{9}$

Nd:YAG (neodymium-doped yttrium aluminum garnet; $\mathrm{Nd}: Y 3 \mathrm{Al5012}$ ) is a crystal that is used as a lasing medium for solid-state lasers. Nd:YAG lasers are optically pumped using laser diodes and typically emit light with a wavelength of $1064 \mathrm{~nm}$, in the infrared. Nd:YAG lasers are one of the most common types of laser, and are used as LLLT for many different applications. ${ }^{10} \mathrm{Nd}$ :YAG lasers have been used to treat chronic periodontitis ${ }^{11}$ and to speed healing of infections around dental implants. ${ }^{12}$

Currently, therapeutic ultrasound is also used primarily in physical medicine. It is a method of Volume 10 Number 2, Dec 2011 
stimulating tissue beneath the skin's surface using sound waves, of a very high frequency, typically between $800,000 \mathrm{~Hz}$ and 2,000,000 Hz. The application of ultrasound to living tissues results in the transmission of mechanical energy in the form of acoustic pressure waves which are transmitted by propagation through the tissue, due to absorption, dispersion or scattering of the wave. ${ }^{13}$ The waves produce forces in tissues equal to micromechanical stresses and strains. It has been established that the micromechanical strains produced by these pressure waves in biological tissues led to biochemical events that regulate fracture healing. ${ }^{14,15}$ Like LLLT, the biological effects of ultrasound can also be categorized as thermal and non-thermal effects. ${ }^{16}$

The purpose of the present investigation is to study the effect of administration of Nd:YAG laser and ultrasound radiation on the histological changes possibly occurring during bone fracture healing process and to investigate whether ultrasound radiation increases or decreases the beneficial effect of Nd:YAG laser.

\section{MATERIALS AND METHODS}

\section{Pilot study}

A cross section through the thigh of 5 male Albino rats (Rattus norvegicus), weighing 250-350 gm and aged 6 months were selected to measure the amount of laser power that pass the skin and other tissues to hit the bone surface. The hairs were shaved, then section blocks containing skin, muscle and bone layers were taken from the thigh and their thickness was measured by vernier. These blocks of tissue were subjected to Nd:YAG (1064 nm wavelength, $135 \mathrm{~mW}$ power)(The laser-Edmund optics worldwide Inc.) from one side of tissue section. Laser power output was measured using power meter that was placed on the opposite side of the section. The measurements were repeated by adding the effect of a CW ultrasound source (50 $\mathrm{mW} \mathrm{cm2}$ intensity, $1 \mathrm{MHz}$ freq. ). The measured output laser power $P$ for each section of thickness $Z$ is used; the mean attenuation coefficient in a certain thickness and wavelength for skin, muscle and bone using the input power $\mathrm{P}^{0}$ for Nd:YAG $135 \mathrm{~mW}$ was calculated using the Beer-Lambert law equation:

$$
p=p_{0}^{*} \exp (-a z)
$$

\section{Experimental Fracture}

Fifteen mature (Rattus norvegicus) rats weighing 250$350 \mathrm{gm}$ were housed in plastic cages, had free access to laboratory chow and water and kept under suitable environmental conditions such as a room temperature that was maintained at about $(24 \pm 2) 0 \mathrm{C}$ and exposed to 12 hour/day light program. Each animal was anesthetized with single intraperitoneal dose of 0.2 $\mathrm{ml}$ of Ketamine (Hyprochloride) $(50 \mathrm{mg} / \mathrm{ml})$ (OUBARI PHARMA-Helepo-Syria) and $0.05 \mathrm{ml}$ Xylazine (Rompun 2\%) (23.32 mg/ml) (OUBARI PHARMA-Helepo-Syria). The left leg was shaved, the skin was cut with sharp razor, the femoral muscle was displaced with forceps, then a partial fracture was made in the lateral as- pect of the upper third of the femur using surgical blade, after that the muscle was replaced and the skin was sutured. A back slap using POP was placed at the site of fracture for all groups to minimize mobilization. The animals were divided into three groups of five rats each. Group 1, was subjected to partial femur fracture and left to be healed without any interference. Group 2, was treated with CW Nd:YAG laser of $1064 \mathrm{~nm}$ wavelength, power of $135 \mathrm{~mW}$ and energyof 16 joulesforduration of 2 minutes; the first dose was given immediately after surgical fracture induction; the subsequent doses were given on days 2 , 3,6 , and then one dose weekly for the next 3 weeks. Group 3, was treated by the addition of the CW ultrasound perpendicular to the laser treatment in group 2, the used ultrasound is of $1 \mathrm{MHz}$ frequency and $50 \mathrm{~mW} / \mathrm{cm} 2$ intensity per session, for 2 minutes on every occasion of laser treatment (The ultrasonic therapy unit-digital Helosonic delux India). Healing site was assessed weekly by X-ray. X-ray showed healing of the site at the 28th day. By the end of 4th week, the rats were sacrificed and the removed femoral bone samples were fixed in fisher fixative for 12 hours, dehydrated through ascending graded alcohols (50\%, $70 \%, 90 \% 100 \%$ ethanol), cleared in two changes of xylene and embedded in paraplast tissue embedding media of 57 oC melting point (Sigma). Serial sections of $5-6 \mu \mathrm{m}$ thickness were stained by Harris's hematoxylin and eosin.

\section{RESULTS}

Table 1 showed the penetrated laser radiation through various tissue thicknesses to reach bone surface. It is clear that the power output of the Nd: YAG laser was increased when ultrasound was added.

Table 1. Power output after passing through a multi-layers tissue consisting of skin, muscle, and one, with and without ultrasound effect.

\begin{tabular}{|c|c|c|}
\hline $\begin{array}{l}\text { Tissue thickness } \\
(\mathrm{mm})(\text { skin }+ \\
\text { muscle }+ \text { bone) }\end{array}$ & $\begin{array}{l}\text { Laser output } \\
\text { power }(\mathrm{mW})\end{array}$ & $\begin{array}{l}\text { Laser + Ultrasound } \\
\text { output power }(\mathrm{mW})\end{array}$ \\
\hline 5.5 & 29 & 41 \\
\hline 6.1 & 27 & 38 \\
\hline 7.5 & 25 & 34 \\
\hline 9.5 & 23 & 30 \\
\hline
\end{tabular}

Histological Results

Group 1 (Control group). The present histological observation showed that bone healing was incomplete; disfiguration and disarrangement of Haversian system is observed (Figure $1 \mathrm{~A}$ ). Separation of the bone, and discontinuity was a marked feature in most of the sections (Figure $1 \mathrm{~B}$ ). New bone matrix lying was observed as bone spicules scattered along the site of injury. Some sections showed the presence of bone spicules at the site of injury with incomplete 


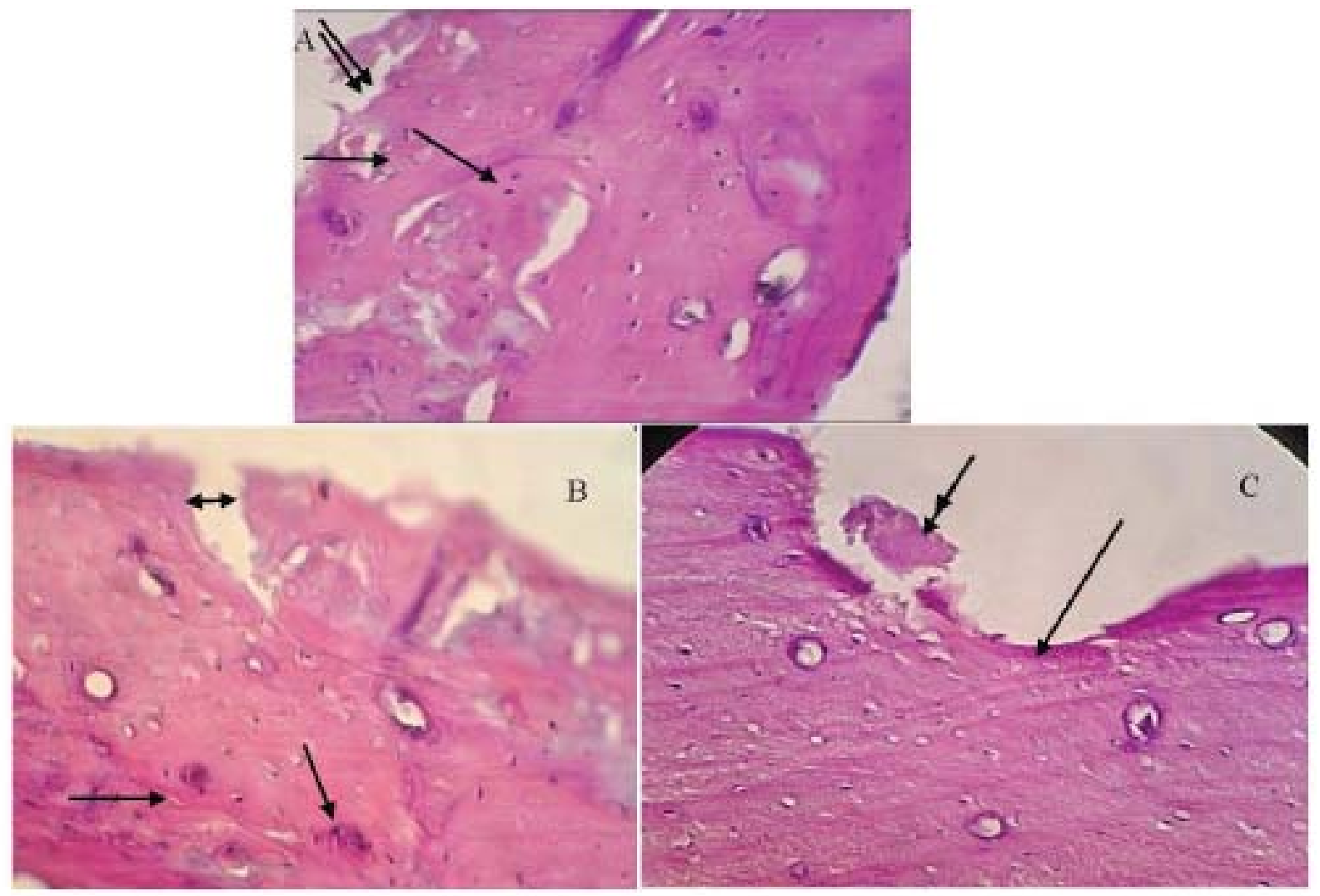

Figure 1A. Sample photomicrographs from bones of control group showing disarrangement of Haversian systems at the site of injury with incomplete bony alignment " $\rightarrow$ " and the periosteum is not yet developed at the site of fracture "Double arrows", Figure 1B. Discontinuity " $\longleftrightarrow$ " of the bone with disarrangement of Haversian systems and bone matrix " $\rightarrow$ ", Figure $1 \mathrm{C}$. Clear defect in the surface of the bone and the periosteum" $\rightarrow$ " with small bone fragments at the site of fractures " $\longrightarrow \longrightarrow$ ". (H\&E stain, original magnification, X 40).

Group 2 (Laser group). The site of healing of the fractured bone showed a complete layer of bone matrix (Figure $2 \mathrm{~A}$ ).
The observed healing area was lacking Haversian system formation, representing Woven bone; there was an irregular cell orientation in the matrix of the healed area (Figure 2B).

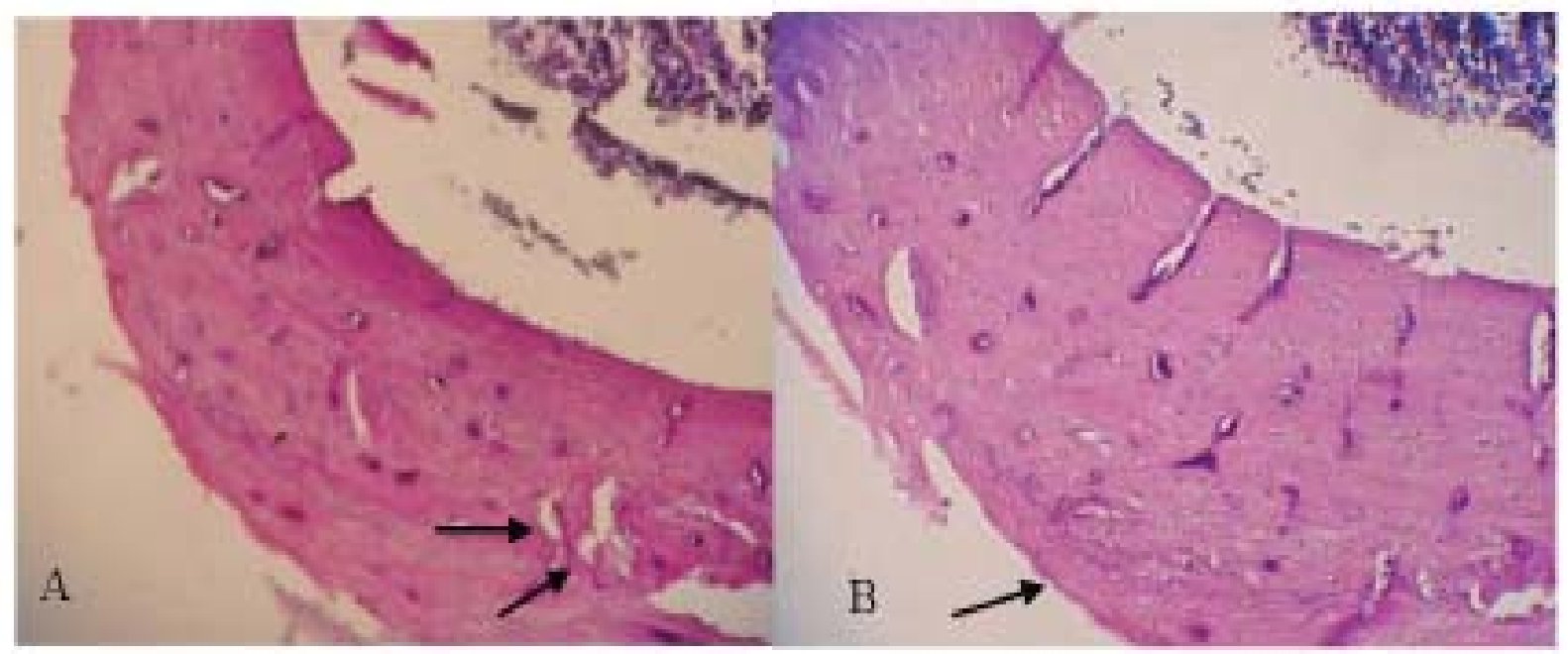

Figure 2. Sample photomicrographs from bones of laser treated group showing the layer of bony material and matrix at the site of injury, but Haversian system is not well developed yet " $\rightarrow$ " $(A)$, the site of injury " $\rightarrow$ " with matrix filling the site and small newly formed Haversian system is not yet developed (B), (H\&E stain, original magnification, X25). 
Group 3 (Laser and UltraGroup 3 (Laser and Ultrasound group). The site of the fractured bone showed a complete healing area at the site of injury. A cross section of the femur showed a regular continuity of the bone surface with regular array of bone marrow inside the bone and a complete layer of periosteum (Figure $3 \mathrm{~A}$ ). The healed area of fracture appeared lightly stained than the adjacent tissue (figure $3 \mathrm{~B}$ ).
This lightly stained area showed a well developed complete periosteum layer of well arranged parallel fibers and cellularity (Figure $3 \mathrm{C} \& \mathrm{D}$ ). A well arranged Haversian systems with well developed lamellae arranged concentrically around a vascular Haversian canal and Volkmann canals were observed (Figures $3 \mathrm{D} \& \mathrm{E}$ ).
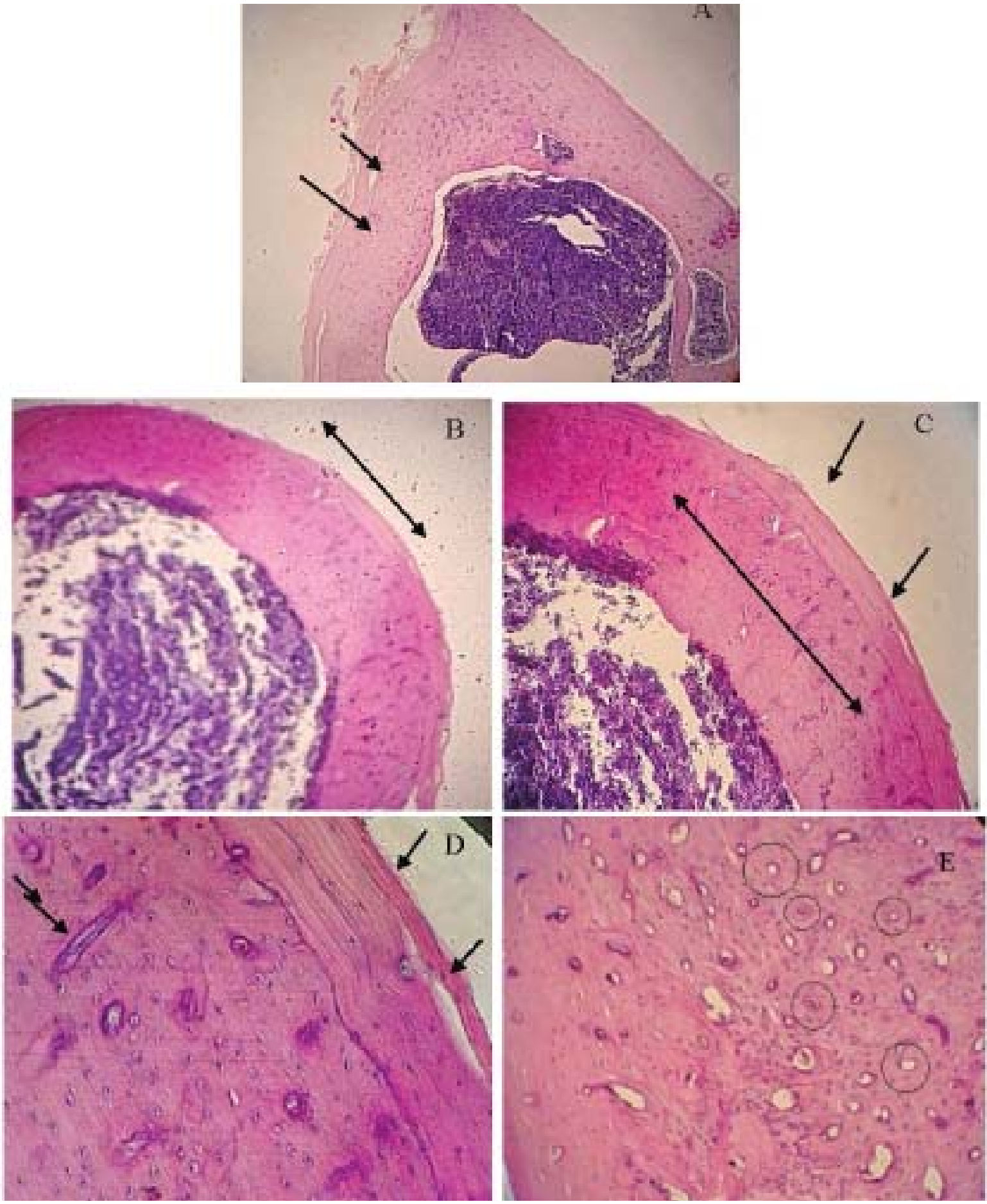

Figure 3. Sample photomicrographs from bones of laser and ultrasound treated group showing a complete bone matrix and a complete layer of periosteum " $\rightarrow$ " $(A)$, completely healed fractured femur, lighter stained area represents the site of injury and healing " $\longleftrightarrow$ " (B), a higher magnification of the area in (B) showing lighter

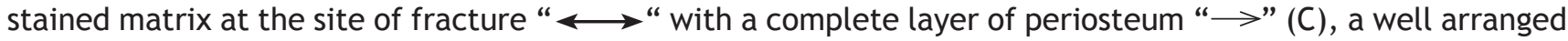
Haversian system, rich vascularity and Volkmann canals " $\longrightarrow$ ", a well developed complete periosteal layer “ $\rightarrow$ " (D), well arranged complete formation of Haversian system at the healed area (E). (H\&E stain, original magnification, “A X10”, “B \& C X25”, “D \& E X40”). 


\section{DISCUSSION}

The use of LLLT in health care has been documented in the literature for more than two decades and has been used in the treatment of a variety of diseases and conditions. The LLLT positive effects on the repair of soft tissues and bone involve metabolic events which may reduce total treatment time and ensure greater patient comfort17 and has a positive effect on the percentage of newly formed bone. ${ }^{18}$

The use of lasers for therapy depends on the interaction of laser beams with tissue; several types of interaction may take place depending on the wavelength of the laser, temporal nature of the beam "continuous wave or pulsed", energy delivered, and special nature of the beam "focused or unfocused". ${ }^{19}$ The interaction of ultrasound with tissues can be also described by reflection that occurs at the tissue surface, absorption by tissues which is often due to coloring agents such as melanin, water and hemoglobin; the absorbed light is generally converted to heat and scattering that causes the light to spread in different directions. All these factors are highly dependent on the wavelength of the incident light. ${ }^{20}$

The present study has shown that the penetration of Nd:YAG laser in the tissue sections "skin, muscle and bone" has increased when the therapeutic ultrasound was added to the applied Nd:YAG. This is due to the effect of the perpendicularly ultrasound application. Ogulu ${ }^{21}$ postulated two mechanisms through which ultrasound modulate the optical field in tissues. First, as light travels the tortuous path of the ultrasonic field, accumulates a phase shift due to multiple reflections from the moving particles of the tissue. Secondly, the index of refraction of the tissue is altered by the pressure field due to the piezooptic effect of the ultrasound, leading to an additional phase change.

Because of its deeper penetration, the Nd:YAG laser was chosen in the present study to illuminate externally an experimentally made partial fracture. The timing of illumination doses was set depending on the fact that the revascularization and the removal of bone debris usually occurs on day 1,2 , and 3 after fracture, while the activation of osteoblast formation occurred on day 6 and $7 .{ }^{16}$

The influence of daily energy doses of He-Ne laser irradiation on the repair of surgically produced tibia damage was investigated in Wistar rats;22 laser treatment was initiated 24 hours after the trauma and continued daily for 7 or 14 days in two groups of nine rats; the course of healing was monitored using morphometrical analysis of the trabecular area; there was a significant increase in the area of new formed trabeculae in tibiae after 7days of irradiation. Trelles and Mayayo ${ }^{23}$ performed histological measurements to determine bone repair with low-power He-Ne laser irradiation in mice; they observed an important increase in vascularization and faster formation of osseous tissue with a dense trabecular net compared to the control group, and concluded that the laser effect might modulate the function of osteocytes, promoting faster metabolism and reaction of bone callus.

In the present study, healing of fractured bones responded to combination of Nd:YAG laser and ultrasound treatment faster than using laser alone. This combination accelerated fracture healing process when complete bone healing was identified in four weeks where the site of healing of the fractured bone showed a regular continuity of bone with regular array bone marrow inside the bone and a complete layer of periosteum, the lamellae of osteons were well arranged around Haversian canals. These observations are in accordance with Trelles and Mayayo ${ }^{23}$ and may be related to the ability of laser irradiation to increase the bone cells activity around the site of the healing area. ${ }^{8}$ Junior et al, ${ }^{24}$ concluded that laser therapy accelerates proliferation of osteoprogenitor cells, enhances osteoblastic calcification and promote bone regeneration. Lirani Galvao et al, ${ }^{25}$ compared the consequences of LLLT and low-intensity pulsed ultrasound (LIPUS) on bone repair; they found that bone histomorphometry revealed a significant increase in osteoblast number and surface, and osteoid volume in the LLLT group, and a significant increase in eroded and osteoclast surfaces in the LIPUS group; they suggested that LIPUS enhanced bone repair by promoting bone resorption in the osteotomy area, while LLLT accelerated this process through bone formation .

Many researchers have discussed the role of using continuous type of laser and its superiority upon pulsed type. The effect of pulse frequency of LLLT on bone nodule formation in rat was studied by Ueda and Shimizuln; ${ }^{26}$ they isolated Osteoblast-like cells from fetal rat calvariae and irradiate them once with a low-energy Ga-Al-As laser in different irradiation modes "continuous and pulsed irradiation". They observed that laser irradiation in all groups significantly stimulated cellular proliferation, bone nodule formation, Alkaline Phosphatase "ALP" activity, and ALP gene expression, as compared with none irradiation group and concluded that the pulse frequency of LLLT constitutes an important factor affecting biological responses in bone formation. In a computerized morphometry; Silva Junior et al. ${ }^{27}$ applied Ga-Al-As laser irradiation on surgical wounds created in the femur of rats and found a significant increase in the mineralization of bone repair at early bone healing in the group subjected to LLLT.

Previous studies reported variable duration of bone healing in fractured bones exposed to various laser's radiation. David et al. ${ }^{28}$ studied the effects of $\mathrm{He}-\mathrm{Ne}$ laser on fracture healing in the tibia of rats and found a gradual increase in healing after 6 weeks of treatment. Chen et al. ${ }^{29}$ studied the effect of a $\mathrm{CO} 2$ laser on bone repair; experimental bilateral mandibular osteotomies were performed in the ramus of 24 rabbits, the calcium and Phosphorus content in 
the irradiated calluses was found to be significantly higher than in the control calluses on the 14th, 21st and 28th post-operation days. Morrone et al. ${ }^{29}$ studied the effect of a GaALAS laser on the osteochondral lesion repair of the knee in rabbits, and found more hyaline-like cells mixed with fibrocartilage in the matrix after 6 and 12 weeks than in the untreated specimens. Enwemeka and Reddy ${ }^{30}$ tested the effect of ultrasound on $632.8 \mathrm{~nm}$ He-Ne laser, and 904 $\mathrm{nm}$ Ga-As laser on healing of experimentally severed and repaired rabbit Achilles tendons as evidenced by biochemical, biomechanical, and morphological indices of healing and found that combinations of the laser with ultrasound stimulation further promote collagen synthesis on through the 14 day period of the study. These variations may be related to species differences and/or to the type of bone used, and type, modes, wave length and episode of laser irradiation. The present investigation has demonstrated that continuous Nd:YAG laser has a healing outcome; and addition of ultrasound to $\mathrm{Nd}$ :YAG laser showed a well developed layer of periosteum. Although structural and cellular healing have occurred after four weeks, it seems that mineralization is still in process and needs further time to complete healing. Further biochemical and ultrastructural studies are needed to study in detail bone healing under the effect of combination of Nd: YAG laser and ultrasound.

\section{CONCLUSION}

NA:YAG Laser therapy has been proved practically to speed up the healing process of bone fractures and the combination of laser and ultrasound in therapy can enhance the healing process of a fractured bone more than laser therapy alone, as the ultrasound increase the depth of laser penetration in tissue.

\section{Acknowledgement}

We are thankful to Mr. Wan Zain $\mathrm{Hj}$. Wan Hassan, the graphic Unit, Kulliyyah of Medicine, IIUM, for his helpful efforts in the organization of the figures of this manuscript.

\section{REFERENCES}

1. Pinheiro ALB, Oliveira MG, Martins PPM, et al. Biomodulatory effects of LLLT on bone regeneration. Laser Ther 2001; 13:73-9.

2. Enwemeka CH, Reddy GK. The biological ef fects of laser therapy and other physical modalities on connective tissue repair processes. Laser Ther 2008; 12:22-30.

3. Djavid GE, Mehrdad R, Ghasemi M, et al. In chronic low back pain, low level laser therapy combined with exercise is more beneficial than exercise alone in the long term: a randomised trial. Aust J Physiother 2007; 53:155-60.

4. Reddy GK, Stehno-Bittel L, Enwemeka CS. Laser photostimulation accelerates wound healing in diabetic rats. Wound Volume 10 Number 2, Dec 2011
Repair Regen 2001; 9:248-55.

5. McLauchlan GJ, Handoll HH. Interventions for treating acute and chronic Achilles tendinitis (Review). Cochrane Database Syst Rev 2009. Published online by John Wiley \& Sons, Ltd. DOI: 10.1002/14651858. CD000232.

6. Ueda Y, Shimizu N. Pulse irradiation of low power laser stimulates bone nodule formation. J Oral Sci 2001; 1:55-60.

7. Tamura K, Hosoya S, Hiratsuka K, et al. Enhancement of mouse CDC46 gene expression in the osteoblast by laser irradiation. Laser Ther 1998; 10:25-31.

8. Barber A, Luger JE, Karpf A, et al. Advances in laser therapy for bone repair. Laser Ther 2001; 13:80-5.

9. Sells B. Laser Physiological and Biological Effects. Vista prints California 2002.

10. Yariv A. Quantum electronics. 3rd edition, Wiley and Sons publishing 1989; 208-211.

11. Cobb CM. Lasers in Periodontics: A Review of the Literature. J Periodont 2006; 77:545-64.

12. Sculean A, Schwarz A, Becker A. Antiinfective therapy with an Er:YAG laser: influence on peri-implant healing. Expert Review of Medical Devices 2005; 2:267-72.

13. Ter Haar C. Basic physics of therapeutic ultrasound. Physiotherapy 1987; 73:110-113.

14. Erikson KR. Laser and Ultrasound in Medicine: A Review. Appears on IEEE Transaction on Lasers and Ultrasonics Symp 1994; 1: 471-84.

15. Johns LD. Nonthermal Effects of Therapeutic Ultrasound: The Frequency Resonance Hypothesis. J Athl Train 2002; 37:293-99.

16. Murray TW, Roy RA. Illuminating sound: Imaging tissue optical properties with ultrasound. Acoustics Today 2007; 3:17-23.

17. Cerqueira A, Silveira RL, Oliveira MG, et al. Bone tissue microscopic finding related to the use of diode laser $(830 \mathrm{~nm})$ in bovine mandible submitted distraction osteogenesis. Acta Cir Bra 2007; 22:92-7.

18. Kreisner P-E, Blaya D-E, Gaião I, et al. Histological evaluation of the effect of low-level laser on distraction osteogenesis $i$ $\mathrm{n}$ rabbit mandibles. Med Oral Patol Oral Cir Bucal 2010; 15:616-8.

19. Rosiak JM, Ulanski P, Kadlubowski S. Conventional and radiation synthesis of polymeric Nano-and Microgel and their possible applications. In Emerging applications of radiation in nanotechnology. Proceedings of a consultants meeting held in Bologna, Italy 2004; March: 99-120.

20. Watson T. Electrotherapy and tissue repair. Sportex Medicine 2006; 29:713-7.

21. Ogulu A. Simulation of Ultrasound Interaction with tissue, an edition from International Centre for Theoretical Physics, Trieste, Italy 1995.

22. Freitas G. Low -Power laser Irradiation Improves Histomorphometrical parameters 
and Bone Matrix Organization during Tibia Wound Healing in Rats. J Photochem Photobiol B: Biology 2003; 70:81-9.

23. Trelles MA, Mayayo E. Bone fracture consolidates faster with low-power laser. Laser Surg Med 1987; 7:36-45.

24. Junior EC, Lopes-Martine AR, Baroni BM, et al. Effect of $830 \mathrm{~nm}$ low-level laser therapy applied before high-intensity exercises on skeletal muscle recovery in athletes. Laser Med Sci 2006; 24:857-63.

25. Lirani-Galvao AP, Jorgetti V, da Silva OL. Comparative study of how low-level laser therapy and low intensity pulsed ultrasound affect bone repair in rats. Photomed Laser Surg 2006; 24:735-40.

26. Ueda Y, Shimizu N. Effects of Pulse Frequency of Low Level Laser therapy (LLLT) On Bone Nodule Formation in Rat Calvarial Cells. J Clin Laser Med Surg 2003; 21:271-7.

27. Silva Junior AN, Pinheiro AL, Oliveira MG, et al. Computerized Morphometric Assessment of the Effect of Low -Level Laser Therapy on Bone Repair: An experimental animal study. J Clin Laser Med Surg 2002; 20:83-87.

28. David R, Nissan M, Cohen I, et al. Effect of low-power He-NE laser on fracture healing in rats. Laser Surg Med 1996; 19:458-63.

29. Chen J, Zhou Y. Effect of low level carbon dioxide laser radiation on biochemical metabolism of rabbit mandibular bone callus. Laser Ther 1989; 1:83-7.

30. Morrone G, Guzzardella G, Torricelli P, et al. Osteochondral lesion repair of the knee in the rabbit after low-power diode GA-AL-AS laser biostimulation: An Experimental Study. Art Cells Blood Subs Immob Biotech 2000; 28:321-336. 
